\title{
Study of Driving and Thermal Stability of Anode-type Ion Beam Source by Charge Repulsion Mechanism
}

\author{
Yunsung Huh $^{\mathrm{a}^{*}}$, Yunseok Hwang ${ }^{\mathrm{a}}$, and Jeha Kim ${ }^{\mathrm{b}^{*}}$ \\ ${ }^{a} R \& D$ Center, Finesolution Co., Ltd., Suwon 16642, Korea \\ ${ }^{b}$ Department of Energy Convergence Engineering, Cheongju University, Cheongju 28503, Korea
}

Received April 27, 2018; revised May 30, 2018; accepted May 31, 2018

\begin{abstract}
We fabricated an anode-type ion beam source and studied its driving characteristics of the initial extraction of ions using two driving mechanisms: a diffusion phenomenon and a charge repulsion phenomenon. For specimen exposed to the ion beam in two methods, the surface impurity element was investigated by using X-ray photoelectron spectroscopy. Upon Ar gas injection for plasma generation the ion beam source was operated for 48 hours. We found a Fe $2 p$ peak 5.4 at. \% in the initial ions by the diffusion mechanism while no indication of $\mathrm{Fe}$ in the ions released in the charge repulsion mechanism. As for a long operation of $200 \mathrm{~min}$, the temperature of ion beam sources was measured to increase at the rate of $\sim 0.1^{\circ} \mathrm{C} / \mathrm{min}$ and kept at the initial value of $27^{\circ} \mathrm{C}$ for driving by diffusion and charge repulsion mechanism, respectively. In this study, we confirmed that the ion beam source driven by the charge repulsion mechanism was very efficient for a long operation as proved by little electrode damage and thermal stability.
\end{abstract}

Keywords: Anode-type ion beam source, Diffusion mechanism, Charge repulsion mechanism, Initial operation characteristics, Electrode damage, Thermal stability

\section{Introduction}

Until now, there are many reports that the use of ion beam sources contributes to improve various thin film performance such as thin film adhesion, thin film density, and electrical characteristics in both pre-treatment and post-treatment, and ion beam assisted processes for thin film heterojunction formation [1-4]. However, in spite of various merits, it is pointed out that the ion beam source can act as an obstacle for the desired process in the case of a long operation that is indispensable for ultra-thin film or many industrial processes. It is because it could cause the generation of impurities, arcing, and particles [5].

After the introduction of the electron bombardment method by a filament in the late 1950s [6-8], the ion beam source of a filament-less end hall type was developed for stable large current ion driving in the mid-1980s. Since such a circular ion beam source emits ions in a cylindrical shape, its use has been limited for specific industrial processes requiring large area processing such as displays, touch screen panels, and building exterior materials, and is still mainly used in thin film processes for optical device. Since then, the Guardian-developed linear source in the late 1990s has become popular as a proper ion beam source among others applicable to large-area industrial processes [9].

*Corresponding author

E-mail:yhuh@finesolution.co.kr, jeha@cju.ac.kr
At present, discussions on grid-less end hall ion beam sources have been actively pursued, such as cooling scheme proposal and charge distribution calculation $[10,11]$, but few studies on anode-type ion beam sources have been reported. In this study, we propose an anodetype ion beam source that is driving initially by both the diffusion mechanism and the charge repulsion mechanism, and investigated it in terms of the impurities and thermal stability in a long operation.

\section{Experiments}

Figure 1 is schematics of the initial plasma generation and ion emission in driving an ion beam source. Figure 1(a)-(c) displays the operation principle of a general ion beam source in which charge emission is started by the diffusion mechanism. For this operation, constant pressure difference is maintained between the inside and the outside of the ion beam source. Simultaneously, a gas for plasma generation is continuously injected into the ion beam source. Figure 1(d) shows the operation principle of an ion beam source in which charge emission is started by the charge repulsion mechanism. In this method, ion plasma is generated by using the process gas in the outside the ion beam device without injecting gas into the ion beam source.

For this experiment, we selected two types of ion beam sources driven by both the diffusion and charge repulsion 


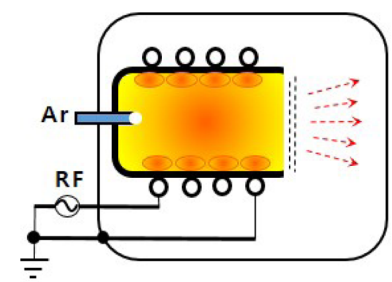

(a)

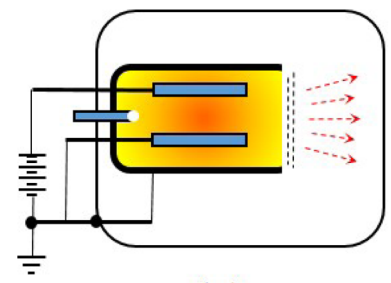

(c)

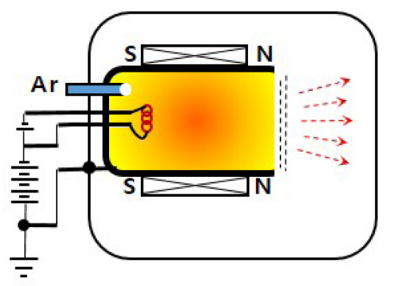

(b)

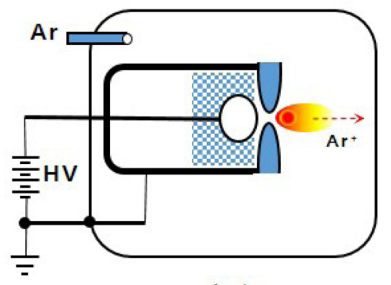

(d)
Figure 1. Operation schematics of ion beam sources; (a) inductively coupled plasma (ICP) type, (b) filament type, (c) capacitively coupled plasma (CCP) type, and (d) repulsion type.

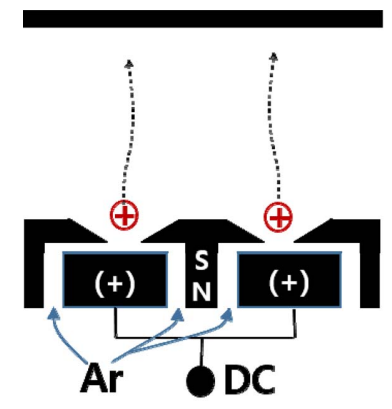

(a)

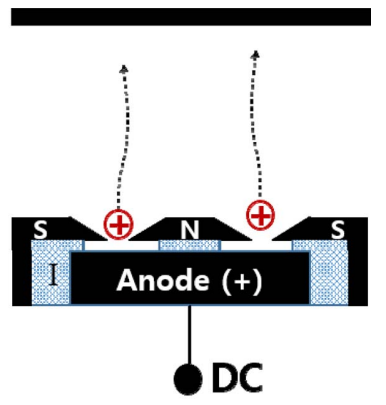

(b)
Figure 2. Schematic drawings for (a) the diffusion mechanism and (b) the charge repulsion mechanism.

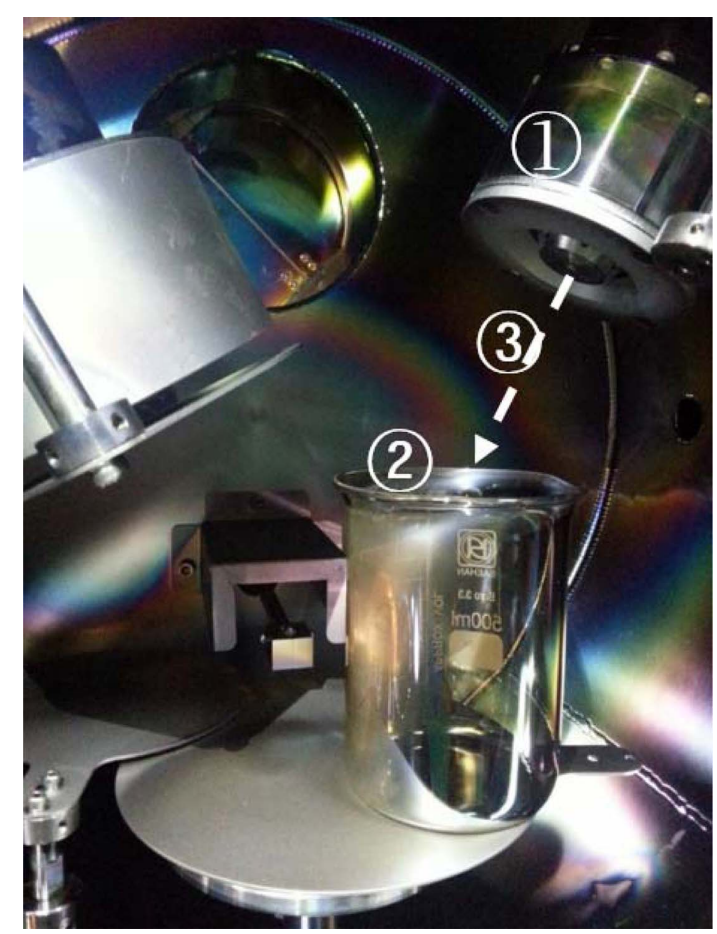

Figure 3. System configuration for the ion beam emission: (1) an ion beam source, (2) a beaker, and (3) the ion beam direction. mechanism as shown in (c) and (d) in Figure 1. Figure 2(a) shows a general ion beam source for injecting the process gas and driven by the diffusion mechanism. Figure 2(b) is an ion beam source (FPG-C100S, Finesolution Co., Korea) driven by a charge repulsion mechanism that used in this experiment $[12,13]$.

In order to study the impurities generation during operation, the ion beam source of the diffusion or charge repulsion mechanism was mounted on the plasma complex machining center $\left(\mathrm{PCMC}^{\mathrm{TM}}\right.$ manufactured by Finesolution Co.) and configured in the layout as shown in Figure 3. The generated ion beam was accelerated toward the inside of a $500 \mathrm{cc}$ Pyrex beaker. A positive voltage of $2,000 \mathrm{~V}$ was applied for plasma generation and ion acceleration, and was continuously operated for 48 hours. At this time, argon (Ar) $5 \mathrm{~N}$ gas was injected for plasma generation, and the Ar pressures while $\mathrm{PCMC}^{\mathrm{TM}}$ operating by the diffusion mechanism and the charge repulsion mechanism were $1.0 \mathrm{mT}$ and $2.0 \mathrm{mT}$, respectively. The chamber pressure was measured using a Pirani gauge, a full range gauge (Pfeiffer), and a capacity diaphragm gauge (MKS). The surface analysis was performed using XPS (K-ALPHA, Thermo Fisher Scientific, UK) to investigate the impurity elements in the ion beam being deposited at the beaker inner surface specimen and we used thermal tape (THERMAX Lever 8, TMC Hallcrest Inc., UK) to measure the temperature of the ion beam source.

\section{Results and Discussion}

Figure 4 is the result of discharge current obtained as a function of the discharge voltage at Ar gas flowing into the ion beam source that was driven by both the diffusion mechanism and the charge repulsion mechanism. The Ar gas was injected at the flow rate of $5 \mathrm{sccm}$ for plasma generation. In the case of the ion beam source driven by the diffusion mechanism, the ion emission was observed satisfactorily at $1.4 \mathrm{mT}$ and $2.0 \mathrm{mT}$, and at $3.0 \mathrm{mT}$, the ion beam showed a critical phenomenon and was not normally released. Comparing to this, however, ions were normally released at $2.0 \mathrm{mT}$ and $3.0 \mathrm{mT}$ in the charge repulsion mechanism. At $1.4 \mathrm{mT}$, the plasma state was weakened. Although the ion beam source driven by the charge repulsion mechanism at relatively low discharge current of below $25 \mathrm{~mA}$ as shown in Figure 4(a), it was driven stably in the region where the gas pressure was as high as above $3.0 \mathrm{mT}$. In general, the discharging current includes various loss currents so that its value is hardly representing the performance of the ion beam source. When driving the ion source as a function of discharge voltage by the charge repulsion mechanism we obtained more stable operation within the relative discharge gradient of \pm 0.02 as seen in Figure 4(b).

Figure 5 is the XPS spectra obtained from the inner surface of a specimen of beaker. Figure 5 shows XPS 


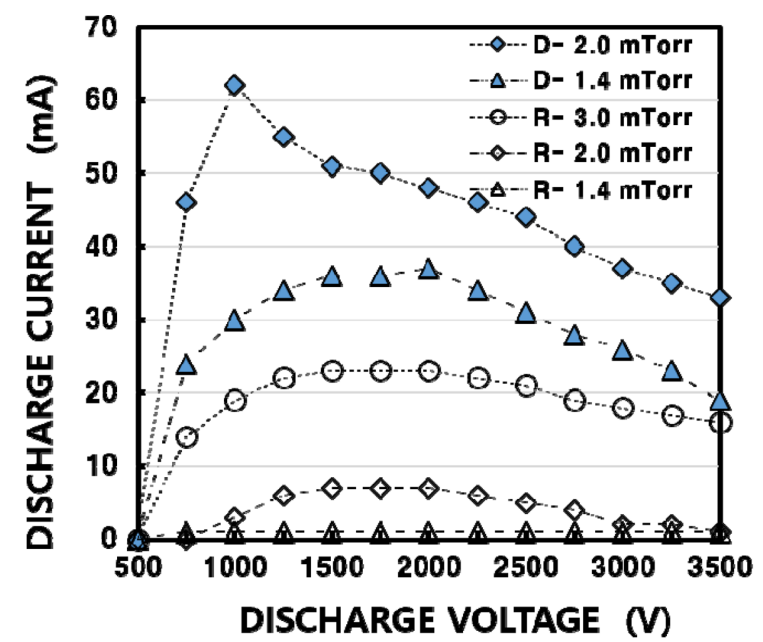

(a)

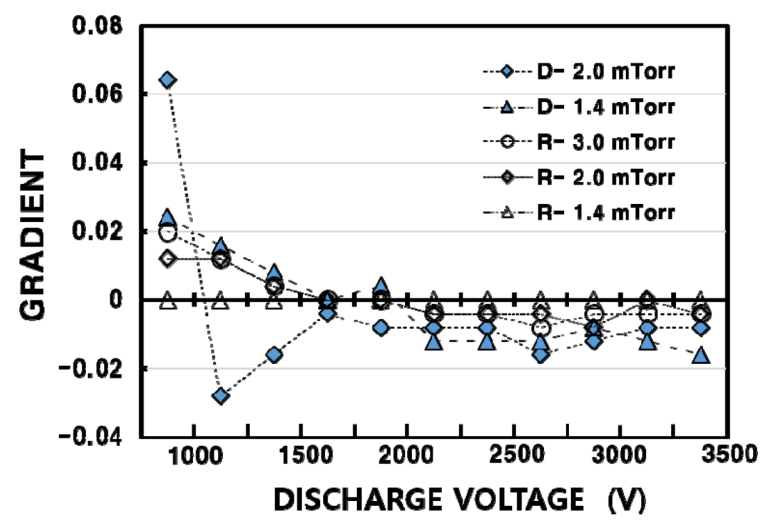

(b)

Figure 4. Discharge current vs. discharge voltage; (a) at various chamber pressures and (b) the change gradient (dI/ dV) rate. ' $D$-' represents diffusion and ' $R$-' for charge repulsion.

spectra obtained from the specimen of beaker: (a) without treatment (reference), (b) treated by the diffusion mechanism, and (c) treated by the charge repulsion mechanism. In Figure 5, there were many elements of $\mathrm{Si}$, $\mathrm{Na}, \mathrm{O}$ and $\mathrm{C}$ of $\mathrm{SiO}_{2}$ and $\mathrm{Na}_{2} \mathrm{O}$ detected from the beaker. In the specimen treated with the ion beam by the diffusion mechanism the Fe element $(\mathrm{Fe} 2 \mathrm{p}, \mathrm{BE}=710.2 \mathrm{eV})$ was detected as in Figure 5(b). In contrast to this, no signal was measured from the specimen surface treated with the charge repulsion mechanism as in Figure 5(c). The Fe element was believed to originate from the cathode electrode when the ions accelerate outward due to the gas pressure difference. The detected Fe element was estimated to be 5.4 at. $\%$ as in the inside detail of Figure 5(b).

This value represents the total sum of the elements on the surface and qualitatively shows the amount occupied by the Fe $2 p$ peak. Therefore, the element of 5.4 at $\%$ can be regarded as a new change due to impurities introduced from outside. The initial plasma and the etching of the slit area of the magnetic pole (cathode) of the ion beam source

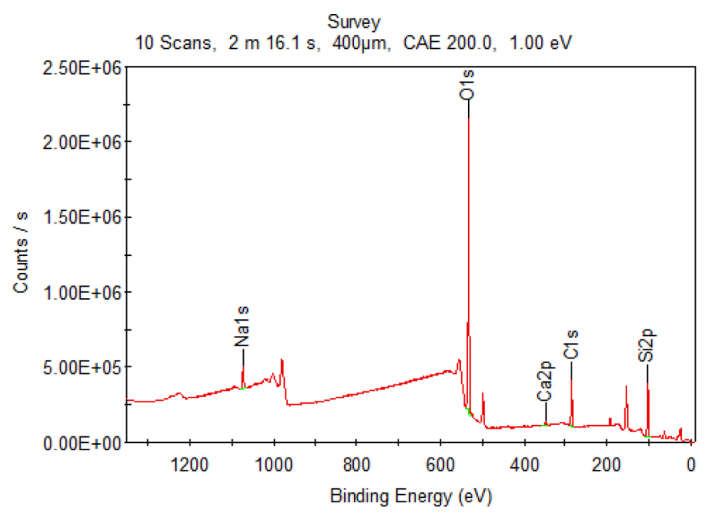

(a)

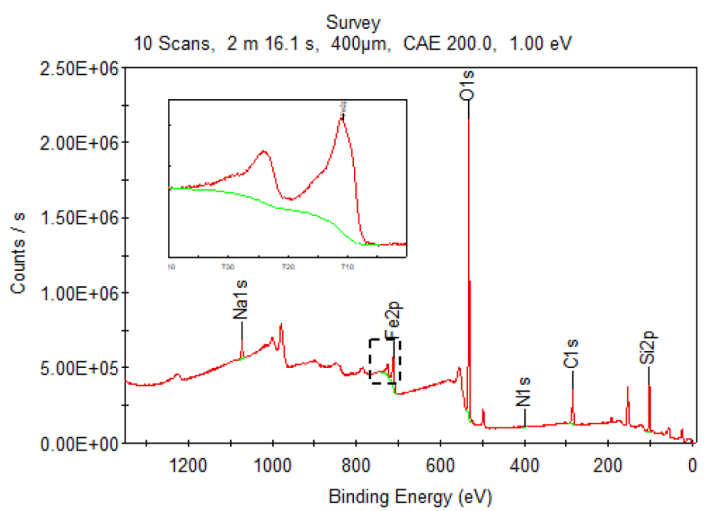

(b)

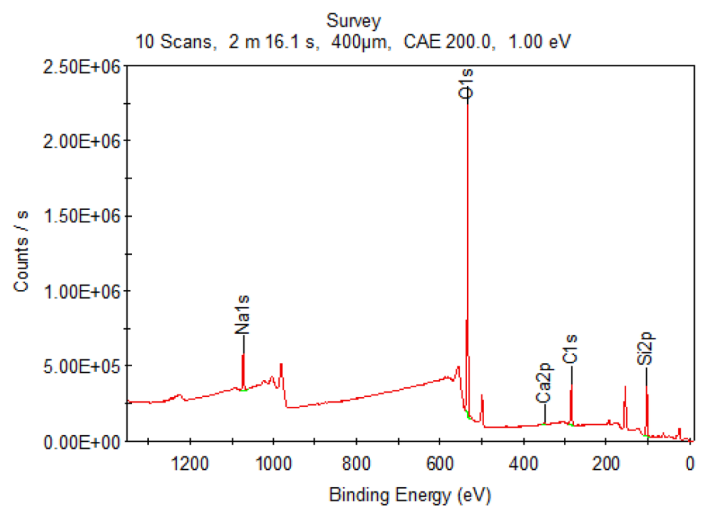

(c)

Figure 5. XPS spectra obtained from the specimen of beaker; (a) no treatment, (b) treated by the diffusion mechanism, and (c) treated by the charge repulsion mechanism.

are commonly generated in the ion release process and are believed to cause a serious problem to the reliability of the product. Thus, an alternative method for preventing this has been suggested [14]. In this experiment, however, no impurity $(\mathrm{Fe})$ element in the repulsive ion beam source was believed to result from the fact that the Ar gas was absent in the source.

Figure 6 shows the results of the heat generation characteristics of the ion beam source for a long operation. To measure the exothermic temperature, we applied a piece 


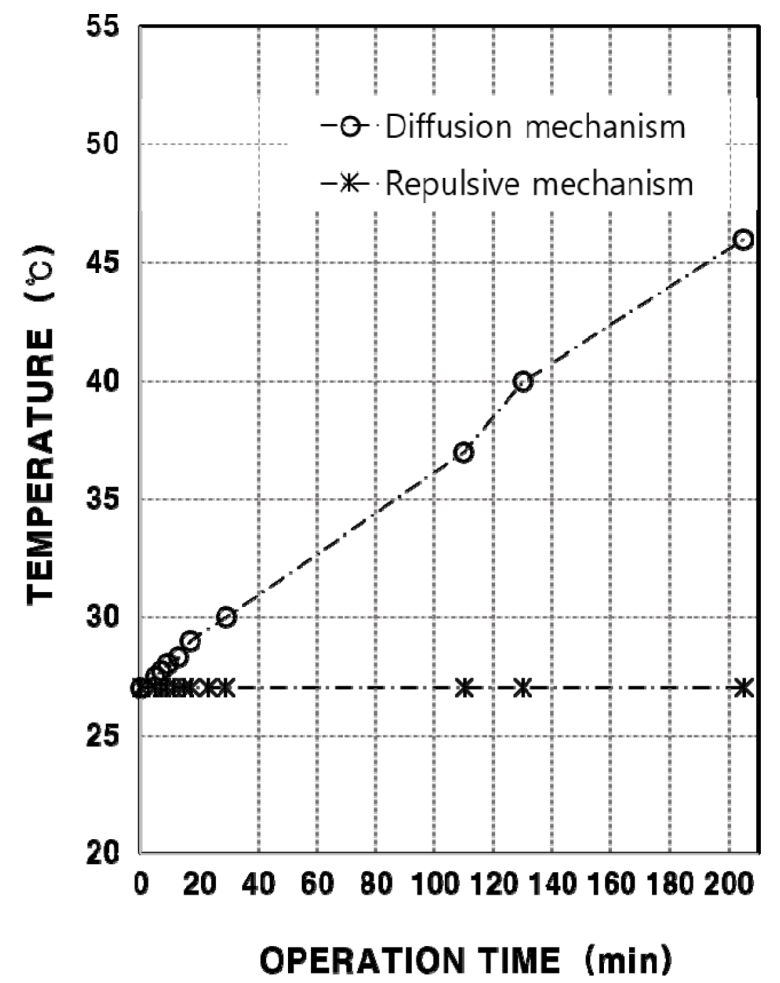

Figure 6. Heat generation of ion beam sources vs. operation time.

of temperature tape to the outside surface of each ion beam source driven by the diffusion and the charge repulsion mechanism, and the temperature was recorded as a function of the driving time.

From this measurement, the ion beam source driven by the charge repulsion mechanism was maintained at the initial temperature $\left(27^{\circ} \mathrm{C}\right)$ in spite of the long time driving. However, in the case of the ion beam source driven by the diffusion mechanism, we observed that the heat gradually was accumulated so that the temperature was increased at the rate of about $0.1{ }^{\circ} \mathrm{C} / \mathrm{min}$. Body heating was attributed to the fact that ions collided with the electrode surface during operation. Thus, it would be inevitable that the etching of the electrode surface was accompanied as a result of collision.

In Figure 2(b) for an ion beam source driven by the charge repulsion mechanism, the anode electrode is surrounded by an insulator and exposed to the outside by the slit interval of the magnetic pole. The ion beam source starts with an initial plasma generation above the exposed anode electrode at the applied voltage of $2,000 \mathrm{~V}$ according to the Child law [15] of the following Eq. (1).

$$
\frac{V_{1}}{V_{2}}=\left(\frac{A_{2}}{A_{1}}\right)^{\delta}
$$

where $A_{1}$ is the counter electrode area facing the anode electrode, and $\mathrm{A}_{2}$ is the exposed area of the anode electrode and $\delta$ is a constant value of 1.5 to 4 depending on the gas type and pressure between the two electrodes. The plasma density is amplified by the electrons which form the closed circuit along the interval between the magnetic poles forming the magnetic field in front of the anode, and the desired voltage can be applied due to the impedance impeding the negative charge (electron) flow. On the other hand, the positive ions in the plasma are accelerated toward the counter electrode due to the charge repulsion force from the moment of ionization due to the electric field of the applied anode voltage. Since the grounding magnetic pole around the anode is close to the potential to the dark space, the initial positive charge is directed toward the specimen located in front of the grounding magnetic pole [10]. Therefore, in the case of the ion beam source driven by the repulsion mechanism the collision between the ion source electrode and the positive charge is fundamentally cut off. It is thus interpreted as an origin of that the impurity and the exothermic phenomenon were not observed in the ion beam source. It is the impurity $(\mathrm{Fe})$ released with the ion beam by the diffusion mechanism that could cause arcing with the particles. Therefore, the ion beam source using the charge repulsion mechanism is more advantageous for securing the stability and durability of the apparatus, in particular, in a long operation.

\section{Summary}

In this study, we tested two anode-type ion beam sources operated by both the diffusion and charge repulsion mechanism and characterized their operation in terms of impurity generation and heat accumulation in a long operation. We found a Fe $2 p$ peak 5.4 at. \% in the initial ions by the diffusion mechanism while no indication of $\mathrm{Fe}$ in the ions released in the charge repulsion mechanism. In addition, the ion beam source driven by the diffusion mechanism was found to cause a temperature rise at the rate of $0.1^{\circ} \mathrm{C} / \mathrm{min}$ while the temperature was kept constantly at $27^{\circ} \mathrm{C}$ for the ion beam source by the repulsive mechanism. It means that the injection of Ar gas for plasma generation inside the ion beam source and the ions by the diffusion mechanism would damage internal electrode seriously for a long operation. In conclusion, the ion beam source by the charge repulsion mechanism provides an efficient way of long operation without generating any impurities in the excellent thermal stability.

\section{References}

[1] H.W. Choi, D.H. Park, J.H. Kim, W.K. Choi, Y.J. Sohn, B.S. Song, J. Cho, and Y.S. Kim, J. Kor. Vac. Soc. 16, 79 (2007).

[2] Y.J. Kim, S.M. Hong, and Y.O. Noh, Polymer 37, 431 (2013).

[3] S.J. Kwon, J. Ins. Elec. Eng. Kor. 44, 26 (2007).

[4] E.S. Cho and S.J. Kwon, J. Kor. Vac. Soc. 22, 26 (2013).

[5] V.V. Zhurin, Surface Engineering 27, 311 (2011).

[6] G.S. Janes and R.S. Lowder, Phys. Fluids 9, 1115 (1966).

[7] H.R. Kaufman, R.S. Robinson, and R.I. Seddon, J. Vac. Sci. Tech. A5, 2081 (1987).

[8] W.C. Kim, K.H. Chung, and B.H. Choi, Rev. Sci. Instr. 65, 1356 
(1994)

[9] V.S. Veerasamy, R.H. Petrmichl, H.A. Luten, and S.V. Thomsen, 45th Annual Technical Conference Proceedings, (Society of Vacuum Coaters, Florida, 2002) pp. 127.

[10] N. Oudini, G.J.M. Hagelaar, J.-P. Boeuf, and L. Garrrigues, J. Appl. Phy. 109, 073310 (2011)
[11] Veeco, Patent No. US7,342,236 B2, Mar. 11 (2008).

[12] Finesolution, Patent No. KR10-1478216, Dec. 24 (2014).

[13] Finesolution, Patent No. US9,269,535 B1, Feb. 23 (2016).

[14] KIMM, Patent No. KR10-1583917, Jan. 4 (2016).

[15] K.S. SreeHarsha, Principles of Physical Vapor Deposition of Thin Films, (Elsevier Science, 2006) pp. 259-365. 